\title{
Remote magnetic targeting of iron oxide nanoparticles for cardiovascular diagnosis and therapeutic drug delivery: where are we now?
}

This article was published in the following Dove Press journal:

International Journal of Nanomedicine

15 July 2016

Number of times this article has been viewed

Michael Bietenbeck'

Anca Florian'

Cornelius Faber ${ }^{2}$

Udo Sechtem ${ }^{3}$

Ali Yilmaz'

'Department of Cardiology and Angiology, ${ }^{2}$ Department

Hospital Münster, Münster,

${ }^{3}$ Division of Cardiology,

Robert-Bosch-Krankenhaus,

Stuttgart, Germany of Clinical Radiology, University
Correspondence: Ali Yilmaz Department of Cardiology and Angiology, University Hospital Münster, Albert-Schweitzer-Campus I, Building AI, 48I49 Münster, Germany

Tel +492518345185

Fax +492518348143

Email ali.yilmaz@ukmuenster.de

\begin{abstract}
Magnetic resonance imaging (MRI) allows for an accurate assessment of both functional and structural cardiac parameters, and thereby appropriate diagnosis and validation of cardiovascular diseases. The diagnostic yield of cardiovascular MRI examinations is often increased by the use of contrast agents that are almost exclusively based on gadolinium compounds. Another clinically approved contrast medium is composed of superparamagnetic iron oxide nanoparticles (IONs). These particles may expand the field of contrast-enhanced cardiovascular MRI as recently shown in clinical studies focusing on acute myocardial infarction (AMI) and atherosclerosis. Furthermore, IONs open up new research opportunities such as remote magnetic drug targeting (MDT). The approach of MDT relies on the coupling of bioactive molecules and magnetic nanoparticles to form an injectable complex. This complex, in turn, can be attracted to and retained at a desired target inside the body with the help of applied magnetic fields. In comparison to common systemic drug applications, MDT techniques promise both higher concentrations at the target site and lower concentrations elsewhere in the body. Moreover, concurrent or subsequent MRI can be used for noninvasive monitoring of drug distribution and successful delivery to the desired organ in vivo. This review does not only illustrate the basic conceptual and biophysical principles of IONs, but also focuses on new research activities and achievements in the cardiovascular field, mainly in the management of AMI. Based on the presentation of successful MDT applications in preclinical models of AMI, novel approaches and the translational potential of MDT are discussed.
\end{abstract}

Keywords: MRI, CMR, magnetic targeting, SPION, myocardial infarction

\section{Introduction}

Magnetic resonance imaging (MRI) provides unique morphological and functional information in the diagnosis and validation of cardiovascular diseases. ${ }^{1}$ Consequently, the clinical role of cardiovascular MRI (CMR) is emerging. In doing so, the diagnostic yield of CMR examinations is often increased by the use of contrast agents (CAs) that are almost exclusively based on gadolinium compounds. Another clinically approved contrast medium is composed of superparamagnetic iron oxide nanoparticles (IONs). ${ }^{2}$ These particles may expand the field of contrast-enhanced CMR, as recently shown in clinical studies focusing on acute myocardial infarction (AMI) and atherosclerosis. ${ }^{3-5}$ Besides their superior safety profile, IONs differ in regard to their biodistribution and biophysical properties, compared to gadolinium-based CAs (GdCAs). The governing mechanism by which IONs influence tissue contrast is different from that of gadolinium complexes, enabling higher relaxation rates, described in detail elsewhere. ${ }^{6,7}$ However, as their effect on transversal relaxation (T2) largely predominates in most cases, they are most efficiently used as T2-accelerators. Thus, along with their particular magnetic 
properties, they create new research opportunities such as remote magnetic drug targeting (MDT). ${ }^{8,9}$ The approach of MDT relies on the coupling of bioactive molecules and magnetic nanoparticles to form an injectable complex. This complex, in turn, can be attracted to and retained at a desired target inside the body with the help of applied magnetic fields. In comparison to common systemic drug applications, MDT techniques promise both higher concentrations at the target site and lower concentrations elsewhere in the body. ${ }^{10}$ Moreover, concurrent or subsequent MRI can be used for noninvasive monitoring of drug distribution and successful delivery to the desired organ in vivo. ${ }^{11}$

This review is in line with recent publications on MDT, $, 12,13$ but in addition to illustrating the basic conceptual and biophysical principles, we particularly focus on new research activities and achievements in the cardiovascular field, mainly in the management of AMI. Based on the presentation of successful MDT applications in preclinical models of AMI, novel approaches and the translational potential of MDT are discussed.

\section{Basic characteristics of iron oxide- based nanoparticles}

IONs exhibit a set of unique properties that are different from that of bulk material, making them object to various biomedical studies. Along with their use in contrast-enhanced MRI (ceMRI), potential translational approaches include cellular/molecular imaging, hyperthermia treatments, and targeted drug delivery. ${ }^{8,14}$

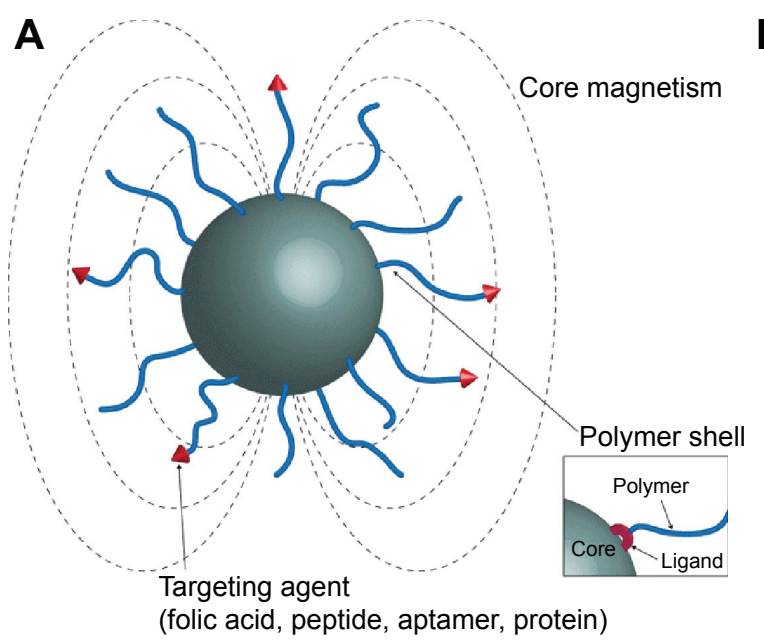

\section{Magnetic properties}

Most ION formulations used in medicine are composed of maghemite and/or magnetite $\left(\gamma-\mathrm{Fe}_{2} \mathrm{O}_{3}\right.$ and $\left.\mathrm{Fe}_{3} \mathrm{O}_{4}\right)$. Both oxides share a similar crystalline structure and are ferri- or ferromagnetic, respectively. Accordingly, their ratio largely determines the particles' overall magnetic properties. ${ }^{15}$ However, when the diameter of these crystals is decreased toward the nanoscale, they become uniformly magnetized at a single domain state and eventually exhibit superparamagnetic behavior when their size is further reduced. ${ }^{16}$ This phenomenon is found in iron oxide crystals that measure less than $35 \mathrm{~nm}$ and is based on the continuous change of their magnetic orientation due to random thermal motion. ${ }^{6}$ As a consequence, the spontaneous formation of a net magnetic field is prevented at room temperature. By contrast, in the presence of an external magnetic field, the magnetic moments align coherently in the direction of the field, forming a net magnetization. This applies to paramagnetic material as well, but in case of superparamagnetic IONs, not only the single magnetic moments but also the net magnetic moments of the uniformly magnetized crystals align to the field, giving rise to a strongly increased magnetic susceptibility.

\section{Particle size and biophysical aspects}

Bare IONs are cytotoxic, tend to agglomerate, and undergo further oxidation, which makes an appropriate coating crucial (Figure 1A). Over recent years, various materials have been proposed for coating, but most ION-colloids intended for medical applications are coated with biocompatible

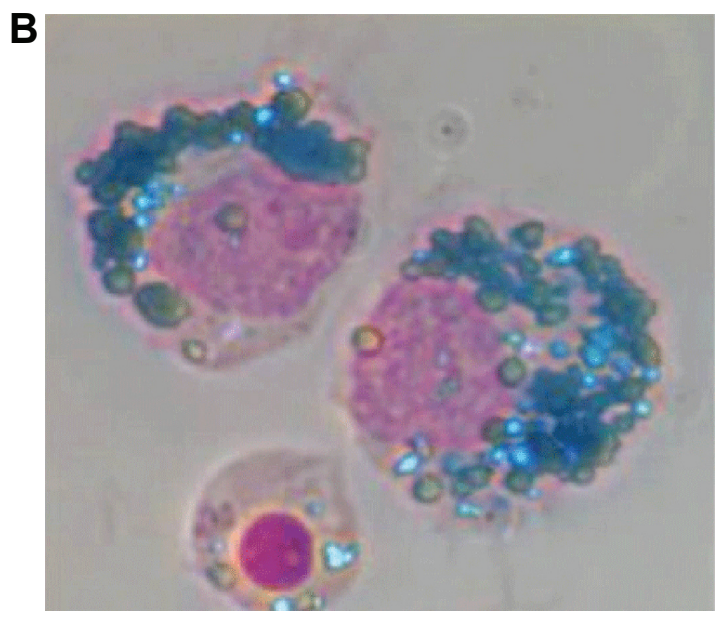

Figure I Schematic drawing of a superparamagnetic iron oxide particle.

Notes: (A) Principle configuration of IONs consisting of a magnetic core and a protective and stabilizing polymer shell. Reprinted by permission from Macmillan Publishers Ltd: NPG Asia Mater; Boyer C, Whittaker MR, Bulmus V, Liu JQ, Davis TP. The design and utility of polymer-stabilized iron-oxide nanoparticles for nanomedicine applications. 2010;2:23-30. Copyright 2010.2 (B) Prussian blue and nuclear fast red staining of cellular SPION uptake (blue) in peripheral blood mononuclear cells. Reprinted from Richards JM, Shaw CA, Lang NN, et al. In vivo mononuclear cell tracking using superparamagnetic particles of iron oxide: feasibility and safety in humans. Circ Cardiovasc Imaging. 20I2;5(4):509-5 I7. Copyright (C) 2012 American Heart Association, Inc. All rights reserved. Promotional and commercial use of the material in print, digital or mobile device format is prohibited without the permission from the publisher Wolters Kluwer. Please contact healthpermissions@wolterskluwer.com for further information. ${ }^{77}$ Abbreviations: IONs, iron oxide nanoparticles; SPION, superparamagnetic iron oxide particle. 
Table I Representative examples for iron oxide-containing contrast agents for MRI with clinical, commercial, and/or considerable experimental use

\begin{tabular}{llll}
\hline Coating & Examples & Size $(\mathbf{n m})$ & Comment \\
\hline Carboxymethyl-dextran & Ferumoxytol (Feraheme ${ }^{\text {TM }}$ ) & $17-31$ & US FDA approved for anemia and in clinical use in the US \\
Carboxydextran & Ferucarbotran (SHU 555A, Resovist $\left.{ }^{\circledR}\right)$ & $45-60$ & US FDA approved; discontinued from clinical use in 2009 \\
Dextran & Ferumoxtran (AMI-227, NCI00 I50, BMS-180549) & $17-21$ & Discontinued after 37 clinical Phase I-III studies in 2010 \\
Dextran & Ferumoxides (Feridex IV $\left.{ }^{\circledR}\right)$ & 100 & Discontinued from clinical use in 2008 \\
Crosslinked dextrans & CLIO-Cy5.5 & $30-35$ & Experimental use \\
Citrate & VSOP-Cl84 & $4-7$ & Experimental use, clinical Phase I study in 2004
\end{tabular}

Notes: Feraheme ${ }^{\text {TM }}$ (AMAG Pharmaceuticals, Waltham, MA, USA), Resovist ${ }^{\circledR}$ (Bayer AG, Leverkusen, Germany), AMI-227 (ARC Machines, Inc., Pacoima, CA, USA). Adapted by permission from Macmillan Publishers Ltd: Nat Mater. Weissleder R, Nahrendorf M, Pittet MJ. Imaging macrophages with nanoparticles. 2014;13:125-138. Copyright 2014.20 Abbreviations: FDA, Food and Drug Administration; MRI, magnetic resonance imaging.

derivatives of dextran (Table 1). ${ }^{17-19}$ In general, the shell is nonmagnetic and thicker than the iron oxide core, and thereby largely defines the final size of the particle. IONs can be classified by their hydrodynamic diameter into 1) superparamagnetic iron oxide particles (SPIONs) with a diameter of 50-250 nm, 2) ultrasmall SPIONs (USPIOs) ranging from 20 to $50 \mathrm{~nm}$, and 3) very small SPIONs (VSPIOs) with a diameter of $<20 \mathrm{~nm}$. However, particle configuration must not be monodisperse in distribution, depending on the synthesis pathway, which in turn directly affects the magnetization and MR imaging properties as further elucidated in the "Distinctiveness of IONs as CAs and drug carriers" section.

After intravenous (IV) injection, the IONs disperse freely in the intravascular compartment and their subsequent biodistribution is principally determined by size, geometry, and chemistry of the coating. Due to their large surface-to-volume ratio, they rapidly adsorb plasma proteins such as opsonins which, in turn, increase their affinity to the mononuclear phagocyte system. According to Weissleder et al, dextrancoated IONs are mainly internalized by the circulating monocytes or tissue-resident macrophages in the liver, spleen, bone marrow, or lymph nodes (Figure 1B). ${ }^{20}$ The organ-specific accumulation is predetermined by the size of the IONs: large SPIONs tend to accumulate in the spleen through mechanical filtering, while smaller VSPIOs are predominantly removed from blood through extravasation and renal clearance. ${ }^{6,21}$ It is noteworthy that cellular uptake is not limited to phagocytosis and monocytes or macrophages. Among other cells, dendritic, cancer, and endothelial cells internalize IONs as well. ${ }^{20,22}$

Apart from the total particle size, the ION's geometry is important in cellular uptake kinetics, as it was shown, for example, for various ellipsoid-shaped particles. ${ }^{23}$ Since recent advances in synthesis pathways allow almost arbitrarily shaped nanoparticles, such as rods, cubes, or husks, with various coating compositions, the geometry will play an increasing role in the design of specific magnetic drug carriers. ${ }^{23,24}$ Furthermore, coatings can be modified in terms of porosity and elasticity, which also affects cellular ION uptake. ${ }^{25,26}$ However, due to the highly specific and partially contradicting data published so far, a conclusive assessment of the shape's specific influence itself or in interplay with other mechanical properties has not been made yet. ${ }^{25,27}$

Following their internalization by macrophages or progenitors, IONs are metabolized by the lysosomal pathway. The iron oxide of the core is sequestered and progressively incorporated into the body's iron store, as shown for ferumoxytol and others. ${ }^{28,29}$ Thereafter, it is eliminated analogously to endogenous iron through the feces. The degradation of the coating is ruled by its specific composition. For example, dextran and its derivatives are degraded by intracellular enzymes and are finally eliminated by renal clearance. ${ }^{30}$

\section{Essential requirements for magnetic targeting of IONs}

The basic concept of (remote) magnetic targeting is fairly straightforward and has been already clinically implemented, for example, in cell separation techniques. Here, cells or molecules are labeled with magnetically responsive nanoparticles that drag along an applied magnetic field gradient and thereby extract the bound target structure from suspension. ${ }^{31}$ However, the adaptation of this principle to an upscaled and more complex (in vivo) system is technically challenging. One major obstacle is the limited interventional depth of permanent magnets that results from the rapid decay of the magnetic field gradients with increasing distance from the magnet. To overcome this problem, various technical setups have been proposed that (among others) include custom-made electromagnets, the use of an arrangement of permanent magnets, the implantation of a magnetizable material, or the design of special gradient coils for conventional MRI scanners. ${ }^{32-36}$

In general, the attractive force exerted on a particle by a magnetic targeting system should equal the hydrodynamic 
drag forces to achieve a sufficiently high attraction/movement against blood flow. If the applied magnetic field is strong enough to completely magnetize the particles, the magnetic force is governed by the equation:

$$
\vec{F}_{m}=M_{s a t} V(\nabla \vec{B})
$$

Accordingly, the magnetization $M_{\text {sat }}$, the volume of the examined material $V$, and the gradient of the magnetic field $\nabla \vec{B}$ are the parameters to be addressed in order to maximize the attracting force. This equation further illustrates why the pure strength of a magnetic field is not entirely decisive for effective targeting. For example, a uniform magnetic field, such as the static field generated in MRI scanners $(\nabla \vec{B}=0)$, will align magnetic moments and magnetically saturate the particles, but does not generate an attractive magnetic force. By contrast, the magnetic field of large permanent magnets may exert attraction at a distance of up to $15 \mathrm{~cm}$; however, this is not the depth limit of MDT. Depending on the particle's features, MDT with a single external bar magnet may exert attraction up to a distance of several centimeters and for moderate flow rates up to $10-20 \mathrm{~cm} / \mathrm{s} .{ }^{17,37}$ It is further assumed that the targeting approaches in femoral and carotid arteries require magnetic flux densities of $200 \mathrm{mT}$ and gradients of up to 8 and $100 \mathrm{~T} / \mathrm{m}$, respectively. ${ }^{37}$ For comparison, current $1.5 \mathrm{~T}$ clinical MR scanners typically provide gradients of up to $50 \mathrm{mT} / \mathrm{m}$. It is noteworthy that a stronger magnetic field/gradient will not necessarily improve the targeting outcome with regard to the aimed biological effect. As shown in a rat model of myocardial ischemia, a $0.3 \mathrm{~T}$ magnet outperformed both 0.15 and $0.6 \mathrm{~T}$ magnets in targeting SPION-labeled stem cells. ${ }^{38}$ Although the $0.6 \mathrm{~T}$ magnet test series showed the highest cell retention, no further increase in the "therapeutic benefit" was observed, which was related to the occurrence of microvascular emboli. Thus, the combination of a magnetic targeting system and the respective ION complex requires careful adaptation to the specific targeting aims and physiological constraints.

Considering the optimization of particle characteristics for MDT, the saturation magnetization of IONs can only be improved slightly by appropriate synthesis pathways. ${ }^{39}$ Other compounds of the same size, such as iron-platinum formulations, exhibit much higher saturation magnetization values, but are not as biocompatible or biodegradable as iron oxides. ${ }^{40}$ Apart from the composition of the core, larger superparamagnetic ION complexes are favorable for targeting approaches in terms of increasing the maximum force per volume, concurrently providing a higher saturation magnetization. ${ }^{15}$ While the magnetic force goes with the particle "volume" $\sim \mathrm{d}^{3}$ (Equation 1), the fluidal drag force scales with the simple "diameter" $\sim$ d. Moreover, larger particles provide higher magnetic relaxation rates, ${ }^{15}$ enabling extremely sensitive cell labeling approaches as demonstrated for superparamagnetic IONs of size $0.9 \mu \mathrm{m}$ by Hinds et al. ${ }^{41}$ In comparison, thousands of V/U/SPIONs would be necessary for a comparable MRI contrast at the same in vivo resolution due to the lower iron content per particle. ${ }^{41}$ However, VSPIOs have previously been used successfully in various cell tracking experiments. ${ }^{42,43}$ It is noteworthy that large IONs show unfavorably decreased blood circulation times for cardiovascular imaging purposes, and thereby also reduce the efficiency of magnetic targeting on the whole and could potentially cause microemboli by unwanted aggregation in small vessels. ${ }^{12,20}$ Shapiro et al also stressed on the potential effects of other particle characteristics such as shape, coating, or flexibility during ION movement through tissue, which need to be considered; however, these settings are not fully elucidated yet. ${ }^{44}$

\section{Distinctiveness of IONs as CAs and drug carriers}

The CAs that are predominantly used in clinical MRI are composed of paramagnetic gadolinium chelates. In principle, such gadolinium complexes shorten the longitudinal relaxation times (T1) of surrounding water protons, whereas their effect on transverse relaxation time (T2) is negligibly low at the recommended concentrations. As a consequence, the tissue containing high concentration of GdCA appears hyperintense in T1-weighted images. A prime example for ceMRI is late gadolinium enhancement imaging in CMR, which enables the distinct delineation of structurally damaged from healthy myocardium. ${ }^{45}$ Following IV administration, GdCA is distributed freely in blood and intestinal space, reaching extracellular equilibrium at approximately 10 minutes after injection, while it is eventually eliminated through renal filtration 1-2 hours later. ${ }^{46}$ During circulation, GdCA cannot pass through healthy cell membranes, but those that are ruptured, for example, during AMI. This represents the biophysical basis for the late gadolinium enhancement imaging technique and enables a rather unspecific detection of damaged areas/tissues. ${ }^{47}$

The use of IONs as CAs may potentially extend the field of ceMRI. In light of the aforementioned characteristics, it is quite obvious that IONs show partly opposite but - according to the application - favorable features in comparison to GdCA: IONs predominantly accelerate T2-relaxation of 
the neighboring tissue, resulting in a local signal void in T2-weighted images, referred to as negative contrast or hypoenhancement. ${ }^{2}$ It is noteworthy that their effect on image contrast varies with particle size, particle size distribution, and aggregation or compartmentalization of IONs, since larger particles or locally coherent particles form single, increased magnetic moments. ${ }^{19}$

Depending on the exact particle size, the half-life of IONs in blood is considerably longer than that of GdCA, for example, 15 hours for ferumoxytol (17-31 nm) (Table 1), providing longer contrast enhancement effects. ${ }^{6}$ Also, they can be safely applied to patients with renal dysfunction, a population that is generally excluded from conventional gadolinium ceMRI. ${ }^{48}$ Moreover, when applied during an ongoing inflammation, iron-laden monocytes are also recruited to the diseased tissue, rendering IONs a potent and promising tracer for inflammation. ${ }^{49}$ In this regard, long blood circulation times are particularly important for cardiovascular applications since they ensure a sufficiently high concentration of free IONs and/or internalized IONs at the target tissue/organ. This characteristic was already shown in clinical trials for myocardial infarction, aortic aneurysms, and carotid plaques. ${ }^{3,4,50,51}$ Furthermore, imaging of iron-laden leukocytes during pathologies such as atherosclerosis, stroke, and various types of cancer is a promising field of current preclinical research, as recently reviewed elsewhere. ${ }^{20}$

IONs have a high sensitivity in MRI, as concentrations in the nanomolar range can be detected and visualized. In addition, they can be conjugated with various biovectors (antibodies, proteins, etc) to increase their specificity, enabling molecular imaging approaches. ${ }^{17}$ IONs can be further conjugated with fluorophores, therapeutics, micro-RNA, and combinations of these, ${ }^{52,53}$ rendering them a versatile platform for targeted drug delivery that can be monitored in vivo by MRI. Common functionalization strategies include covalent bonding or grafting of ligands to the IONs' coating. Further approaches include the encapsulation of bioactive molecules and IONs into vehicles such as liposomes, micelles, or polymer nanoparticles, ${ }^{54}$ and the immobilization of IONs and active ligands onto carbon nanomaterial. ${ }^{55}$ However, when delivery of ION-bound therapeutics is considered, the challenging issue of unwanted/wanted release kinetics in response to internal and/or external (remote) stimuli comes into focus. Potential stimuli for a controlled drug release from the carrier vehicle include changes in $\mathrm{pH}$ and enzyme concentrations or heating of thermosensitive bonds, as comprehensively reviewed by Mura et al. ${ }^{56}$

\section{General considerations regarding simulations of MDT in tumors and cardiovascular diseases}

Most solid tumors comprise areas of impaired oxygen supply that result from rapid tumor growth and associated abnormal angiogenesis. ${ }^{57}$ Disorganized and less dense vascular networks accompanied by temporally unstable blood flow eventually cause these hypoxic microenvironments that are considered to play a central role in the occurrence of, for example, chemoresistance, resistance to cell death, and metastasis. ${ }^{57}$ On the other hand, the abnormally grown tumor vasculature shows enhanced permeability, allowing macromolecules to accumulate in tumor interstitial space, while hampered lymphatic filtration retains them there. ${ }^{58}$ This enhanced permeability and retention effect is an important bridge to many conventional systemic drug therapies in the field of oncology. ${ }^{58}$ Unfortunately, the appearance and severity of both hypoxic zones and the enhanced permeability and retention may vary significantly within and between tumor types and patient populations, rendering systemic IV drug delivery in oncology challenging. ${ }^{57,58}$ In order to increase drug concentration at the target site, magnetically controlled drug targeting can be used. ${ }^{12}$ Notably, Lubbe et al were one of the first to use magnetically controlled drug targeting in humans in the 1990s during therapy of advanced cancers and achieved radically decreased tumor volumes in the absence of significant toxicities, ${ }^{10}$ but since subsequent dose escalation trials revealed poor tumor response, ${ }^{59}$ the focus turned back on preclinical research.

To test and optimize specific magnetic setups, particle formulations, etc, numerical simulation should be carried out before using animal models. However, tumor tissue heterogeneity, varying morphology and volume, as well as unstable blood supply of the abnormal growth do not really allow an accurate and consistent numerical modeling. In contrast, there is substantially lower variation in basic conditions in the cardiovascular field, for example, regarding flow conditions and vascular access to injured myocardium, potentially enabling a more successful estimation of magnetic targeting efficacy that, however, does not necessarily imply a potential drug response or even a beneficial therapeutic outcome.

Nevertheless, for the sake of computability, most models largely simplify the pathological/physiological conditions by simulating an impermeable (branched) tube with preset flow conditions, potentially rendering their calculations applicable to a broader field beyond cardiovascular diseases. The general basis of MDT modeling is the 
comparison of magnetic force to blood drag forces exerted on IONs by calculating the magnetic forces using Maxwell's equations and their effect on particle trajectories in a viscous fluid and laminar flow using Navier-Stokes equations. More recent and comprehensive models also include ION magnetization states below saturation, interparticle forces, more realistic fluid dynamics, diffusion forces, and/or ION concentration effects. In this context, the comprehensive review of Nacev et al nicely illustrates a detailed approach for mapping out the IONs' behavior during MDT experiments. ${ }^{8}$ By means of normalizing various physiological and physical parameters to characteristic scales, for example, flow velocity to maximum centerline velocity, the domains of three key numbers/parameters were used to define prototypical behaviors: velocity-dominated, magnetic force-dominated, and boundary layer formation (at early, intermediate, and steadystate time snapshots) after ION application. The ordering of these characteristics determines the probability of magnetically induced accumulation of the evaluated particles. While in case of boundary layer formation, direct particle retention is presumed, the circulation time is crucial for magnetic targeting in the magnetic force-dominated regime. To validate their approach, Nacev et al used observations from past in vitro and animal experiments that were consistent with their model predictions. ${ }^{8}$ Thus, the approach of these authors is a promising starting point for the conception of specific MDT setups using a single magnetic source.

Another recent publication focused on the multiphysical simulation of steering magnetic nanoparticles in an asymmetric bifurcation. ${ }^{60}$ The authors modeled the three-dimensional motion of ION clusters influenced by hematocrit, shear rate, and an applied magnetic force. Based on their comprehensive calculations, they concluded that clusters of particles are needed to magnetically displace the particles to a sufficient extent and that in case of a bifurcation, this will be most successful when equal magnetic gradients (up to $10 \mathrm{~T} / \mathrm{m}$ ) are applied toward the branch and opposite to the streamwise direction. ${ }^{60}$ In a similar approach, Haverkort et al simulated MDT in a geometry mimicking the left coronary artery (LCA), which was calculated based on angiographies from healthy patients. ${ }^{61}$ Their model further included rather large iron-carbon particles $(0.25-4 \mu \mathrm{m})$ and a superconducting cylindrical magnet, which was positioned in different orientations at a distance approximating the distance from the LCA to the patient's chest $(\sim 5 \mathrm{~cm})$. In the LCA model, the capture efficacy was found to increase in a linear fashion with particle diameter, depending on the position of the magnet. Although their models included various simplifications, such as restricted fluid dynamics, that potentially underestimate the capture efficacy of smaller particles, ${ }^{61}$ the presented calculations and results state strong reasons for further investigations of MDT and the additional incorporation of physiologically/ clinically relevant parameters such as the orientation/position of the magnet in regard to the target tissue.

\section{Preclinical applications of magnetic targeting in cardiovascular diseases}

Treatment of cardiovascular diseases with gene or stem cell formulations holds promise to improve myocardial tissue recovery and therapeutic outcome. Although various delivery routes and compounds were examined, such approaches are still limited by poor accumulation and retention of the therapeutic at the target site, impairing their therapeutic potential and translation into the clinic. ${ }^{62}$ To the best of our knowledge, Cheng et al were the first to address this limitation by the magnetically enhanced delivery of cardiac-derived stem cells (CDCs) in a preclinical setting (Table 2 briefly summarizes the studies presented hereafter). ${ }^{63}$ They cultured male CDCs along with fluorophore-carrying superparamagnetic microspheres (SPM) of size $0.9 \mu \mathrm{m}$ that were applied to female rats subjected to AMI. Ischemia was induced by permanent ligation of the left anterior descending coronary artery

Table 2 Preclinical studies on magnetic drug targeting

\begin{tabular}{lllllll}
\hline $\begin{array}{l}\text { Year and } \\
\text { study }\end{array}$ & $\begin{array}{l}\text { Model } \\
\text { (\# animals) }\end{array}$ & $\begin{array}{l}\text { ION size } \\
(\mathbf{n m})\end{array}$ & $\begin{array}{l}\text { Therapeutic } \\
\text { moiety }\end{array}$ & $\begin{array}{l}\text { Complex } \\
\text { size }(\mathbf{n m})\end{array}$ & $\begin{array}{l}\text { Magnetic field } \\
\text { strength }(\mathbf{T})\end{array}$ & $\begin{array}{l}\text { Increased retention } \\
\text { versus controls }\end{array}$ \\
\hline $2010^{63}$ & LAD lig (88 rats) & 900 & CDCs & - & 1.3 & $>3 \times$ \\
$2012^{64}$ & LAD I/R (82 rats) & 900 & CDCs & - & 1.3 & $>5-6 \times$ \\
$2012^{65}$ & LAD lig (I0I rats) & 100 & hVEGF gene & $300-600$ & 1.0 & $>4 \times$ \\
$2013^{68}$ & LAD I/R (108 rats) & $45-60$ & MSCs & 500 & 0.6 & $3.87 \times$ \\
$2013^{69}$ & LAD I/R (90 rats) & $45-60$ & MSCs & 500 & 0.6 & $2.87 \times$ \\
$2014^{70}$ & LAD I/R (58 rats) & $17-31$ & CDCs & - & 1.3 & $>3 \times$ \\
$2014^{11}$ & LAD I/R & $17-31$ & BMSCs & $80-110$ & 1.3 & $>3 \times$ \\
$2015^{38}$ & LAD I/R (2I7 rats) & $45-60$ & MSCs & - & $0.15,0.3,0.6$ & $1.45 \times, 2.76 \times, 3.86 \times$ \\
\hline
\end{tabular}

Abbreviations: BMSCs, bone marrow-derived stem cells; CDCs, cardiac-derived stem cells; I/R, ischemia/reperfusion; LAD lig, permanent ligation of left anterior descending artery; MSC, mesenchymal stem cell; ION, iron oxide nanoparticle. 
(LAD). Then, starting with the onset of intramyocardial CDC injection, a $1.3 \mathrm{~T}$ circular magnet was placed approximately $1 \mathrm{~cm}$ above the heart apex for 10 minutes during the surgical procedure. In doing so, slight discoloration of adjacent tissue was visible to the naked eye, indicating the retention of SPMlabeled CDCs against coronary washout. The retention of SPM-labeled CDCs was confirmed by histology 24 hours later. Semiquantitative fluorescence imaging revealed that substantially more cells were spread to the lung and spleen within the subsets of rats injected with non- or SPM-labeled CDCs in the absence of a magnet, as compared to those rats that received labeled cells and underwent additional magnetic targeting. Subsequent polymerase chain reaction (PCR) analyses for the sex-determining SRY gene revealed a threefold higher CDC engraftment into the myocardial tissue of rats in the magnetic targeting group. Accordingly, the authors concluded that magnetic targeting is capable of attenuating the washout of SPM-labeled CDCs from the injection site and that only 10 minutes of magnetic targeting is sufficient to increase short-term CDC engraftment significantly.

These initial results and conclusions were confirmed in a rat model of LAD ischemia/reperfusion by the same group. ${ }^{64}$ They employed a similar experimental setup, but additionally evaluated different magnet application times (up to 6 hours) after intracoronary injection of labeled cells. These pilot tests showed that CDC retention increased only to a lesser extent with prolonged magnet application duration, whereas the rats' condition became worse with longer open-chest intervals. Consequently, the magnet application time was kept at 10 minutes for subsequent experiments, accepting smaller increases of attracted CDCs than would have been possible with longer duration of magnet application. Nevertheless, in comparison to control groups, magnetic targeting of labeled CDCs led to (up to) six times higher retention after 24 hours.

In 2012, Zhang et al examined the capability of magnetic field-assisted gene delivery in a rodent model of permanent LAD ligation. ${ }^{65}$ The study was designed as a continuation of previous trials that had already successfully proven the principle in healthy mice. ${ }^{66,67}$ During the course of surgery, either a $1.1 \mathrm{~T}$ magnet or a ceramic bar of identical shape was fixed to the blanched myocardium. The IV-injected gene formulation was composed of adenoviral vectors that were conjugated with superparamagnetic nanobeads, forming particles of size 300-600 nm. Viral vehicles were loaded with genes of human vascular endothelial growth factor or fluorescent moieties. Immunohistochemical staining performed 2 and 7 days after the induction of ischemia indicated successful magnetic attraction of labeled complexes to myocardial segments next to the magnet. Subsequent quantitative analyses revealed significantly increased expression of VEGF in hearts assigned to the magnet-positive group as compared to controls, 7 days after myocardial infarction. In addition, gene expression was significantly lower in the liver tissue of magnet-positive group, indicating that magnetically enhanced delivery is indeed capable of modifying in vivo gene distribution and expression throughout the body (Figure 2).

In 2013, two preclinical studies were published by Huang et al, evaluating the efficacy of stem cell injections into the left ventricular cavity and the left retrograde cardiac vein through a transjugular catheter. ${ }^{68,69}$ In both settings, a similar model of $\mathrm{LAD}$ ischemia/reperfusion was employed in female rats. The therapeutic complexes consisted of male mesenchymal stem cells that were labeled with ferucarbotran (45-60 nm) (Table 1) and fluorophores. To improve cell retention, a cylindrical magnet with a calculated flux density of approximately $0.6 \mathrm{~T}$ was placed in the direct vicinity of the injection site for 10 minutes during reperfusion. In vivo T2-weighted MRI was performed 24 hours after the injection, followed by ex vivo fluorescence imaging and PCR analysis of the explanted hearts. Interestingly, no total regional signal void was seen in the acquired MR images, but relative hypointensities were observed at the anterior left ventricle wall in groups treated with labeled cells. Among the population of rats that was treated with additional magnetic targeting, the degree of hypoenhancement was significantly increased, indicating a higher accumulation of ferucarbotran. Moreover, in comparison to controls and

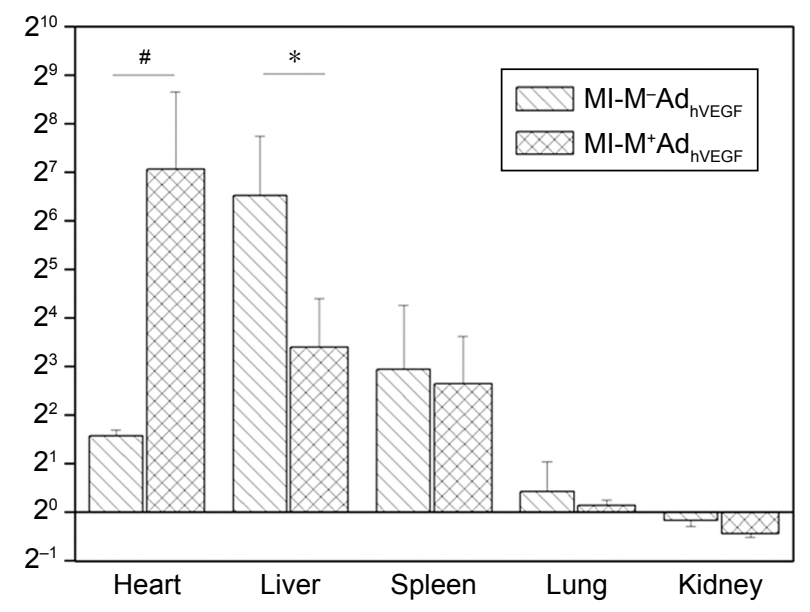

Figure 2 Organ distribution of a hVEGF-encoding ION gene formulation following intravenous injection in rats with $\mathrm{Ml}$ and with $\left(\mathrm{M}^{+}\right)$or without $\left(\mathrm{M}^{-}\right)$magnetic targeting. $* P<0.05,{ }^{*} P<0.05$. Reproduced with permission from Zhang $\mathrm{Y}, \mathrm{Li} \mathrm{W}, \mathrm{Ou} \mathrm{L}$, et al. Targeted delivery of human VEGF gene via complexes of magnetic nanoparticleadenoviral vectors enhanced cardiac regeneration. PLoS One. 2012;7(7):e39490..$^{65}$

Abbreviations: hVEGF, human vascular endothelial growth factor; ION, iron oxide nanoparticle; M, magnet; MI, myocardial infarction. 
depending on the delivery route, a 2.7- to 3.4-fold higher fluorescence signal was detected in hearts subjected to magnetically enhanced delivery of cells. Analogously, PCR analyses revealed 2.8- to 3.8-fold more male mesenchymal stem cells at the target site when a magnet was applied.

While the studies presented so far employed comparable large IONs, Vandergriff et al were the first to use the smaller USPIO ferumoxytol (17-31 nm) along with a fluorophore for labeling and magnetically attracting the CDCs. ${ }^{70}$ After induction of LAD ischemia/reperfusion in female rats, male CDCs were administered as intracoronary injection during an additional temporary occlusion of the aorta. At the same time, a 1.3 T magnet was superimposed to the open injection site for 10 minutes. T2*-weighted MR images acquired after 24 hours revealed enlarged areas of hypoenhancement in the magnetic targeting group in comparison to controls (Figure 3A). This was confirmed by semiquantitative fluoroscopic imaging, while quantitative PCR could determine the effect of magnetic targeting to a triplication of retained CDCs (Figure 3B). It is noteworthy that the injection of CDC-ferumoxytol conjugates did not induce iron overload, nor did the magnetically enhanced accumulation of the complexes result in microemboli as tested in preliminary studies. ${ }^{70}$

Only recently, Cheng et al reported on a new particle formulation termed "magnetic bispecific cell engager", composed of functionalized ferumoxytol particles (Table 1). ${ }^{11}$ Two different antibodies were attached to its coating in order to bind to the injured myocardium and capture the circulating endogenous stem cells. The complex was prepared for IV injection and tested in a rodent model of ischemia/ reperfusion. ${ }^{11}$ In order to enrich these "matchmakers" at the infarcted tissue, in the first place, a 1.3 T magnet was placed over the left thoracic region for 10 minutes after the onset of reperfusion. In T2*-weighted MR images acquired 24 hours later, relative hypoenhancement in the left ventricular anterior wall was significantly higher in the magnet-positive group as compared to controls. Moreover, analysis of confocal microscopic images performed another 4 weeks later revealed an almost doubled number of recruited endogenous stem cells after magnetic targeting. ${ }^{11}$
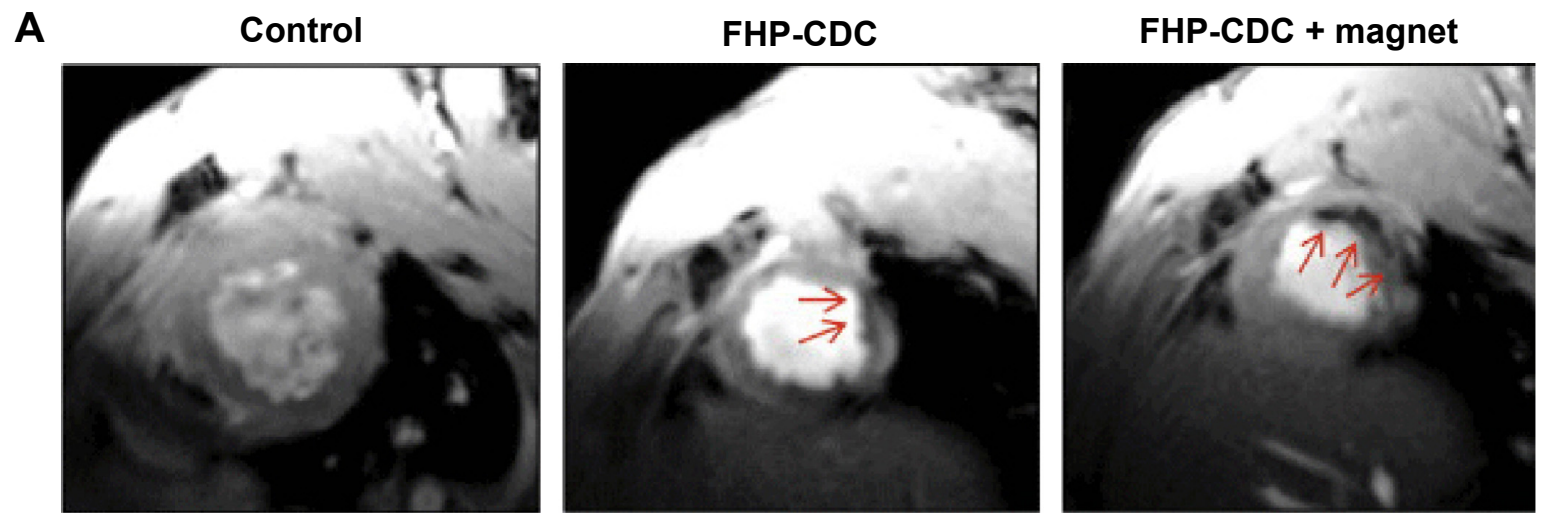

B

Cell retention in the heart by qPCR

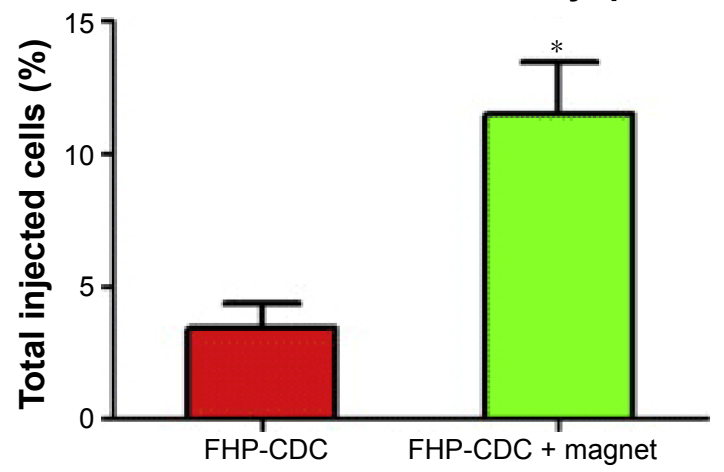

Figure 3 External magnetic targeting in an animal model of myocardial infarction.

Notes: (A) T2*-weighted MR images acquired 24 hours after the induction of myocardial infarction, indicating the increased retention of ION-labeled stem cells with the help of an external magnet (FHP-CDC, signal hypointensities marked by red arrows), which was confirmed by (B) quantitative PCR. $* P<0.05$. Reprinted from Biomaterials, 35(30), Vandergriff AC, Hensley TM, Henry ET, et al. Magnetic targeting of cardiosphere-derived stem cells with ferumoxytol nanoparticles for treating rats with myocardial infarction, 8528-8539. Copyright 2014; with permission from Elsevier. doi: 10.1016/j.biomaterials.2014.06.031. ${ }^{70}$

Abbreviations: FHP-CDC, ferumoxytol-heparin-protamine labeled cardiac-derived stem cells; ION, iron oxide nanoparticle; MR, magnetic resonance; qPCR, quantitative polymerase chain reaction. 
In the most recent study, Shen et al compared different magnetic intensities for in vivo targeting of in vitro iron-labeled stem cells. ${ }^{38}$ The cells obtained from male rats were labeled with ferucarbotran $(45-60 \mathrm{~nm})$ and/or fluorophores. After 90 minutes of LAD ligation and 20 minutes of reperfusion, the labeled cells were injected into the left ventricle cavity of female rats. Magnetic targeting was performed either with $0.15 \mathrm{~T}, 0.3 \mathrm{~T}$, or $0.6 \mathrm{~T}$ magnets that were positioned in close vicinity of the exposed heart. In comparison to controls, magnetic targeting led to a 1.45 -, 2.76-, and 3.86-fold increased cell retention rate as quantified by PCR. The magnetic intensity and the number of retained cells correlated in a positive and linear fashion. Histological staining and fluoroscopic imaging, however, revealed that higher magnetic strengths not only induced higher retention rates but also formed an increased number of microvascular emboli. The authors argued that such emboli were responsible for the lack of therapeutic benefit in the $0.6 \mathrm{~T}$ group when compared to the 0.3 T cohort. $^{38}$

\section{Promising future approaches in iron oxide-based diagnosis and therapy}

Most cardiovascular diseases are characterized by an inflammatory response, initiating complex cardiac repair mechanisms. The accurate assessment and subsequent modulation of such inflammatory conditions is of major interest in risk stratification, therapeutic decision making, and therapy monitoring. In this context, ceMRI using IONs opens up new vistas. Their additional and unique diagnostic value was already proven in the settings of AMI, acute myocarditis, and acute cardiac transplant rejection in both animal models and human trials (Figures 4 and 5)..$^{3,4,20,71,72}$ Hence, the use of ION-based CA can be easily implemented in existing CMR protocols. To increase their specificity, IONs can be functionalized to bind certain target structures, as shown for early cardiomyocyte apoptosis in rats, for instance. ${ }^{73}$ Apart from the use of pure ION formulations, such functionalized ION approaches are highly promising, given that appropriate ION-based compounds get legal approval as diagnostic CAs. Ferumoxytol, the only remaining ION complex that is still Food and Drug Administration approved for IV application in the US, was recently withdrawn from the European Union market due to commercial reasons. Nevertheless, in view of their unique features, we expect ION formulations to play an important role in future noninvasive imaging, improved therapeutics, and combinations of both.

Regarding theranostic approaches, the ION-antibody functionalization strategy proposed by Cheng et al seems very

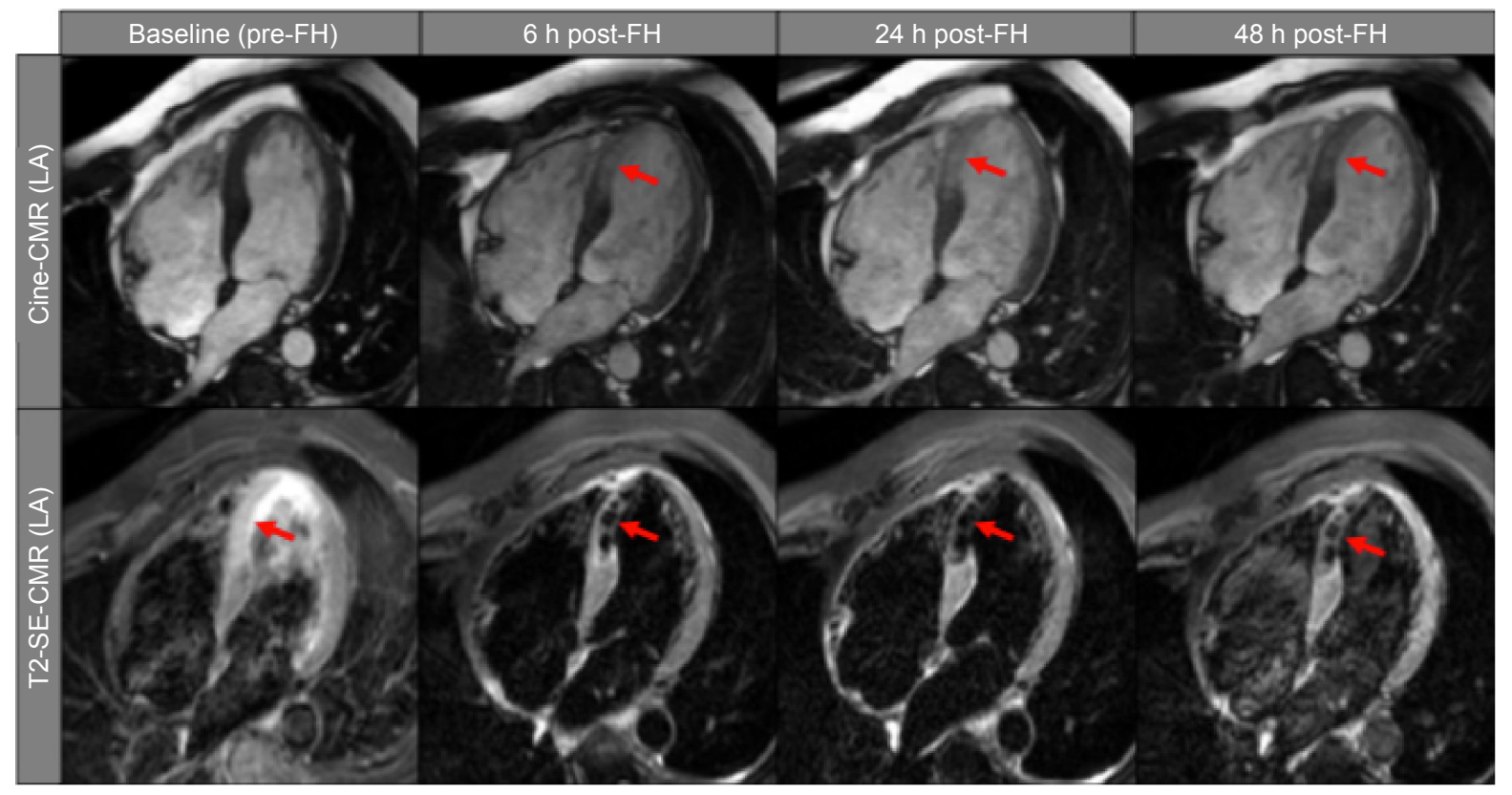

Figure 4 Exemplary cardiac magnetic resonance images in the long-axis view of a study patient with a septal myocardial infarction who underwent pre-Feraheme ${ }^{\mathrm{TM}}$ (AMAG Pharmaceuticals, Waltham, MA, USA) (baseline) and post-FH (after 6, 24, 48 hours) cardiovascular magnetic resonance imaging studies, respectively.

Notes: The first line shows cine-CMR images at different time points with proof of hyperenhancement in the septal wall at 6-48 hours post-FH (red arrows). The second line shows T2-weighted short-tau inversion recovery spin-echo images at different time points with proof of hypoenhancement in the septal wall at 6-48 hours post-FH (red arrows). Reproduced with permission from Yilmaz A, Dengler MA, van der Kuip H, et al. Imaging of myocardial infarction using ultrasmall superparamagnetic iron oxide nanoparticles: a human study using a multi-parametric cardiovascular magnetic resonance imaging approach. Eur Heart J. 20I3;34(6):462-475, by permission of Oxford University Press on behalf of the European Society of Cardiology. ${ }^{4}$

Abbreviations: cine-CMR, cine-cardiovascular magnetic resonance; h, hours; FH, Feraheme; LA, long-axis. 

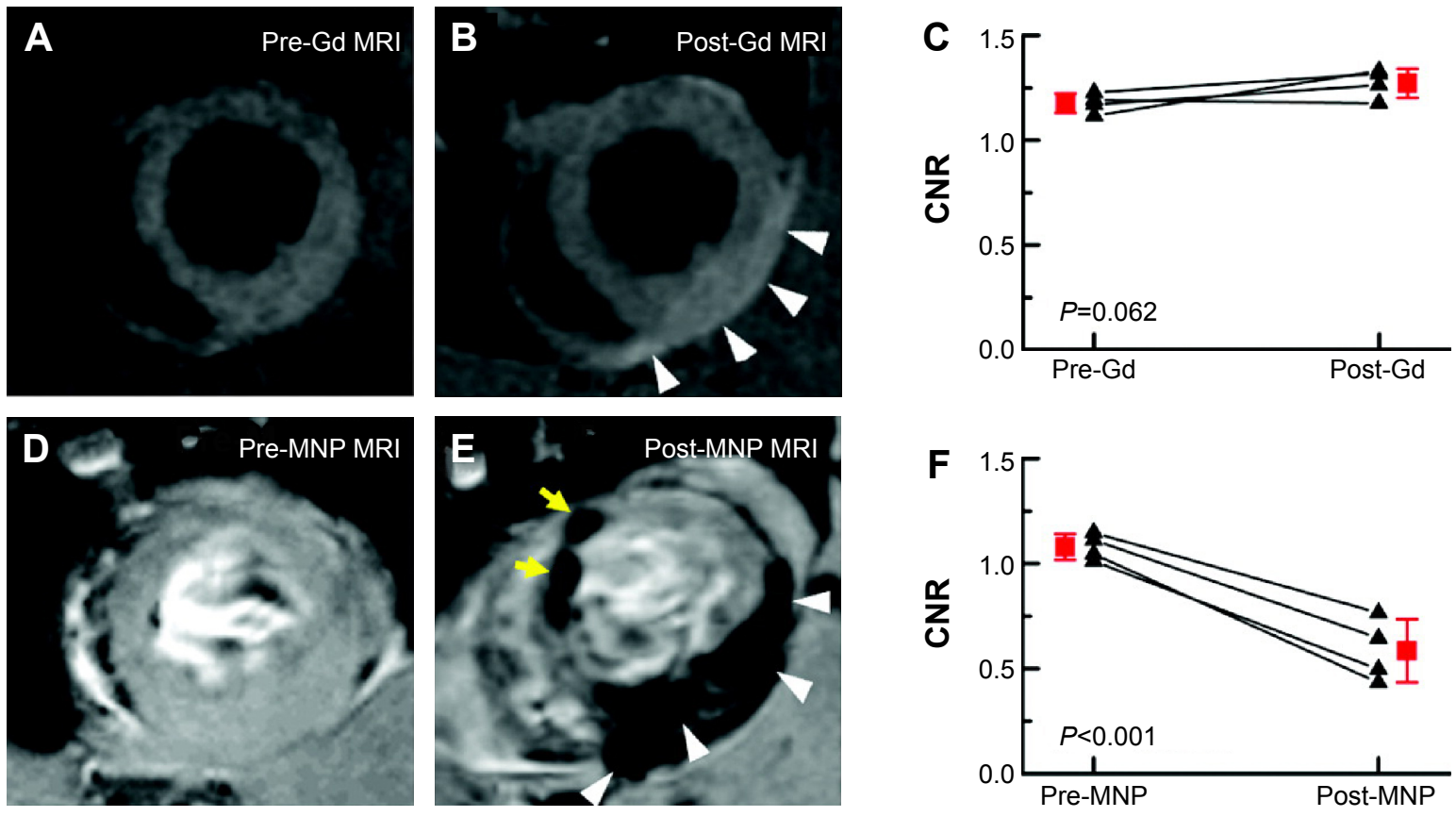

Figure 5 MRI images acquired in short-axis view in a rat model of acute myocarditis.

Notes: (A-B) TI-weighted Gd contrast-enhanced images and corresponding CNR diagram (C); (D-E) T2*-weighted acquisitions before and after MNP application ( 85 nm in-house synthesized) and corresponding CNR diagram (F). Inflammation-associated contrast enhancement is visualized with the white arrowheads (B and $\mathbf{E})$ in the left ventricular wall. Images of MNP-treated rats show significantly larger changes in CNR determined in left ventricular wall. Yellow arrows mark in (E) the inflammatory spots not found in post-Gd images but present in post-MNP acquisitions. ( $\mathbf{C}$ and $\mathbf{F}$ ) black indicates individual values and red indicates mean value. Republished with permission of American Heart Association, from Noninvasive assessment of myocardial inflammation by cardiovascular magnetic resonance in a rat model of experimental autoimmune myocarditis; Moon H, Park HE, Kang J, et al; I25(21), 20I2; permission conveyed through Copyright Clearance Center, Inc. ${ }^{71}$

Abbreviations: CNR, contrast-to-noise ratio; Gd, gadolinium; MNP, magnetic nanoparticle; MRI, magnetic resonance imaging.

promising. ${ }^{11}$ As presented in detail in the previous section, such double-conjugated IONs act as a "matchmaker" between the injured tissue and a therapeutic moiety, while the entire complex is still highly responsive to magnetic fields. These features, in turn, can be exploited for both diagnostic MRI and successful MDT. In our opinion, such strategies hold great translational potential and may accelerate the development of novel disease-specific therapeutic options.

However, the main unsolved challenge for clinical MDT approaches - apart from the aforementioned drug release issues - is the delivery of appropriate magnetic gradients that are required to navigate a sufficiently high number of nanocomplexes, even against the blood flow. This issue becomes even more difficult when the cardiovascular system with its related high arterial flow rates is concerned. In a simplistic comparison, hydrodynamic drag forces occurring in large vessels typically outnumber magnetic attractive forces. Thus, simple handheld magnets will not be useful beyond animal models. However, we believe that strategies combining ION-laden cells/vehicles with either optimized Halbach arrays, arrangements of electromagnets, or clinical MRI scanners are promising for future MDT applications. Halbach arrays consist of a special layout of permanent magnets that amplifies the magnetic field on one side while the field is almost canceled out at the other face of the array. Using numerical optimization, Sarwar et al simulated a 36-element array that creates five times greater magnetic forces at $10 \mathrm{~cm}$ depth than a benchmark magnet of the same volume and material. ${ }^{33}$ If this arrangement could be designed with a light weight and as a mobile solution to enable longer magnet exposure times, for example, at bedside, long circulating ION formulations could be attracted more efficiently to the desired tissue/organ. A more sophisticated method that relies on a dynamic feedback control scheme of eight electromagnets positioned around the patient was proposed in 2012 by Nacev et al. ${ }^{74}$ During the course of simulation, however, perfect real-time imaging was assumed to assess the current position of a SPION assembly, which in turn was fed back into the control algorithm that orchestrates the magnets.

In order to accelerate clinical implementation of MDT, setups and ION formulations that had already received regulatory approval would be preferable. In this regard, Muthana et al recently published a pivotal paper on the potential of MRI scanners to steer ION-laden cells into one or more target tissues. ${ }^{75}$ Using a $300 \mathrm{mT} / \mathrm{m}$ gradient system, they were able to navigate the macrophages loaded with SPIONs and oncolytic 
viruses to prostatic tumors in mice. Spatial gradients were applied in a pulsed fashion for a total of 1 hour, resulting in a significantly increased SPION-laden macrophage uptake in tumorous tissue that could be translated into a significant increase in therapeutic efficacy when compared to controls. These results are particularly encouraging since new generations of clinical MRI scanners and coil systems are capable of producing similar gradient strength in deep tissue. ${ }^{76}$

\section{Conclusion}

Nanotechnology and nanoscience will definitely improve the future diagnosis and treatment of cardiovascular diseases. Valuable progress has been made in the development of nanoparticle synthesis pathways and functionalization strategies, but resultant formulations are still limited to basic and preclinical research. Their diagnostic as well as therapeutic yield can be further improved with the help of magnetic targeting systems, exploiting their superparamagnetic nature. These systems have to provide appropriate magnetic gradients to increase the ION/ nanocomplex concentration at the site of interest. In our view, either specially adapted clinical MR scanners or arrangements of permanent magnets hold the potential to fulfill this demand in cardiovascular settings in the near future.

\section{Disclosure}

The authors report no conflicts of interest in this work.

\section{References}

1. American College of Cardiology Foundation Task Force on Expert Consensus Documents; Hundley WG, Bluemke DA, Finn JP, et al. ACCF/ACR/AHA/NASCI/SCMR 2010 expert consensus document on cardiovascular magnetic resonance: a report of the American College of Cardiology Foundation Task Force on Expert Consensus Documents. $J$ Am Coll Cardiol. 2010;55(23):2614-2662.

2. Boyer C, Whittaker MR, Bulmus V, Liu JQ, Davis TP. The design and utility of polymer-stabilized iron-oxide nanoparticles for nanomedicine applications. NPG Asia Mater. 2010;2:23-30.

3. Alam SR, Shah AS, Richards J, et al. Ultrasmall superparamagnetic particles of iron oxide in patients with acute myocardial infarction: early clinical experience. Circ Cardiovasc Imaging. 2012;5(5):559-565.

4. Yilmaz A, Dengler MA, van der Kuip H, et al. Imaging of myocardial infarction using ultrasmall superparamagnetic iron oxide nanoparticles: a human study using a multi-parametric cardiovascular magnetic resonance imaging approach. Eur Heart J. 2013;34(6):462-475.

5. Tang TY, Howarth SP, Miller SR, et al. The ATHEROMA (Atorvastatin Therapy: Effects on Reduction of Macrophage Activity) Study. Evaluation using ultrasmall superparamagnetic iron oxide-enhanced magnetic resonance imaging in carotid disease. J Am Coll Cardiol. 2009;53(22): 2039-2050.

6. Krishnan KM. Biomedical nanomagnetics: a spin through possibilities in imaging, diagnostics, and therapy. IEEE Trans Magn. 2010;46(7): 2523-2558.

7. Strijkers GJ, Nicolay K. Relaxivity of nanoparticles for magnetic resonance imaging. In: Sattler KD, editor. Handbook of Nanophysics: Nanomedicine and Nanorobotics. Boca Raton, FL, USA: CRC Press; 2010.
8. Nacev A, Beni C, Bruno O, Shapiro B. The behaviors of ferro-magnetic nano-particles in and around blood vessels under applied magnetic fields. J Magn Magn Mater. 2011;323:651-668.

9. Pankhurst QA, Connolly J, Jones SK, Dobson J. Applications of magnetic nanoparticles in biomedicine. J Phys D Appl Phys. 2003;36(13): $167-181$.

10. Lubbe AS, Bergemann C, Riess H, et al. Clinical experiences with magnetic drug targeting: a phase I study with $4^{\prime}$-epidoxorubicin in 14 patients with advanced solid tumors. Cancer Res. 1996;56(20): 4686-4693.

11. Cheng K, Shen D, Hensley MT, et al. Magnetic antibody-linked nanomatchmakers for therapeutic cell targeting. Nat Commun. 2014; 5:4880.

12. Pankhurst QA, Thanh NTK, Jones SK, Dobson J. Progress in applications of magnetic nanoparticles in biomedicine. J Phys D Appl Phys. 2009;42(22):224001.

13. Connell JJ, Patrick PS, Yu Y, Lythgoe MF, Kalber TL. Advanced cell therapies: targeting, tracking and actuation of cells with magnetic particles. Regen Med. 2015;10(6):757-772.

14. Laurent S, Dutz S, Häfeli UO, Mahmoudi M. Magnetic fluid hyperthermia: focus on superparamagnetic iron oxide nanoparticles. $A d v$ Colloid Interface Sci. 2011;166(1-2):8-23.

15. Kolhatkar AG, Jamison AC, Litvinov D, Willson RC, Lee TR. Tuning the magnetic properties of nanoparticles. Int J Mol Sci. 2013;14(8): 15977-16009.

16. Lu AH, Salabas EL, Schüth F. Magnetic nanoparticles: synthesis, protection, functionalization, and application. Angew Chem Int Ed Engl. 2007; 46(8):1222-1244.

17. Laurent S, Saei AA, Behzadi S, Panahifar A, Mahmoudi M. Superparamagnetic iron oxide nanoparticles for delivery of therapeutic agents: opportunities and challenges. Expert Opin Drug Deliv. 2014; 11(9): 1449-1470.

18. Roohi F, Lohrke J, Ide A, Schutz G, Dassler K. Studying the effect of particle size and coating type on the blood kinetics of superparamagnetic iron oxide nanoparticles. Int J Nanomedicine. 2012;7: 4447-4458.

19. Tsourkas A, Josephson L. Magnetic nanoparticles. In: Weissleder R, editor. Molecular Imaging: Principles and Practice. 1 st ed. Shelton, CT, USA: PMPH-USA; 2010:523-541.

20. Weissleder R, Nahrendorf M, Pittet MJ. Imaging macrophages with nanoparticles. Nat Mater. 2014;13:125-138.

21. Gupta AK, Gupta M. Synthesis and surface engineering of iron oxide nanoparticles for biomedical applications. Biomaterials. 2005; 26(18):3995-4021.

22. Thorek DL, Tsourkas A. Size, charge and concentration dependent uptake of iron oxide particles by non-phagocytic cells. Biomaterials. 2008;29(26):3583-3590.

23. Champion JA, Mitragotri S. Role of target geometry in phagocytosis. Proc Natl Acad Sci U S A. 2006;103(13):4930-4934.

24. Doshi N, Mitragotri S. Designer biomaterials for nanomedicine. $A d v$ Funct Mater. 2009;19(24):3843-3854.

25. Anselmo AC, Zhang M, Kumar S, et al. Elasticity of nanoparticles influences their blood circulation, phagocytosis, endocytosis, and targeting. ACS Nano. 2015;9(3):3169-3177.

26. Mitragotri S, Lahann J. Physical approaches to biomaterial design. Nat Mater. 2009;8(1):15-23.

27. Hillaireau H, Couvreur P. Nanocarriers' entry into the cell: relevance to drug delivery. Cell Mol Life Sci. 2009;66(17):2873-2896.

28. Demolis P, Laslop A, Melchiorri D, Ruiz S, Sancho-Lopez A. CHMP assessment report: Rienso. EMA/CHMP: 59-68. April 19, 2012.

29. Reimer P, Balzer T. Ferucarbotran (Resovist): a new clinically approved RES-specific contrast agent for contrast-enhanced MRI of the liver: properties, clinical development, and applications. Eur Radiol. 2003;13(6):1266-1276.

30. Wang AZ. Nanoparticles for cancer diagnosis and therapy. In: Webster TJ, editor. Safety of Nanoparticles: From Manufacturing to Medical Applications. New York, USA: Springer; 2008;209-236. 
31. Miltenyi S, Muller W, Weichel W, Radbruch A. High gradient magnetic cell separation with MACS. Cytometry. 1990;11(2):231-238.

32. Alexiou $C$, Diehl D, Henninger $P$, et al. A high field gradient magnet for magnetic drug targeting. IEEE Trans Appl Supercond. 2006;16(2): $1527-1530$.

33. Sarwar A, Nemirovski A, Shapiro B. Optimal halbach permanent magnet designs for maximally pulling and pushing nanoparticles. J Magn Magn Mater. 2012;324(5):742-754.

34. Polyak B, Fishbein I, Chorny M, et al. High field gradient targeting of magnetic nanoparticle-loaded endothelial cells to the surfaces of steel stents. Proc Natl Acad Sci U S A. 2008;105(2):698-703.

35. Fu A, Wilson RJ, Smith BR, et al. Fluorescent magnetic nanoparticles for magnetically enhanced cancer imaging and targeting in living subjects. ACS Nano. 2012;6(8):6862-6869.

36. Riegler J, Wells JA, Kyrtatos PG, Price AN, Pankhurst QA, Lythgoe MF. Targeted magnetic delivery and tracking of cells using a magnetic resonance imaging system. Biomaterials. 2010;31(10):5366-5371.

37. Voltairas PA, Fotiadis DI, Michalis LK. Hydrodynamics of magnetic drug targeting. J Biomech. 2002;35(6):813-821.

38. Shen Y, Liu X, Huang Z, et al. Comparison of magnetic intensities for mesenchymal stem cell targeting therapy on ischemic myocardial repair: high magnetic intensity improves cell retention but has no additional functional benefit. Cell Transplant. 2015;24(10):1981-1997.

39. Wu W, Wu Z, Yu T, Jiang C, Kim WS. Recent progress on magnetic iron oxide nanoparticles: synthesis, surface functional strategies and biomedical applications. Sci Tech Adv Mater. 2015;16(2):23501.

40. Figuerola A, Di CR, Manna L, Pellegrino T. From iron oxide nanoparticles towards advanced iron-based inorganic materials designed for biomedical applications. Pharmacol Res. 2010;62(2):126-143.

41. Hinds KA, Hill JM, Shapiro EM, et al. Highly efficient endosomal labeling of progenitor and stem cells with large magnetic particles allows magnetic resonance imaging of single cells. Blood. 2003;102(3): 867-872.

42. Strobel K, Hoerr V, Schmid F, Wachsmuth L, Loffler B, Faber C. Early detection of lung inflammation: exploiting T1-effects of iron oxide particles using UTE MRI. Magn Reson Med. 2012;68(6):1924-1931.

43. Hoerr V, Tuchscherr L, Huve J, et al. Bacteria tracking by in vivo magnetic resonance imaging. BMC Biol. 2013;11:63.

44. Shapiro B, Kulkarni S, Nacev A, Muro S, Stepanov PY, Weinberg IN. Open challenges in magnetic drug targeting. Wiley Interdiscip Rev Nanomed Nanobiotechnol. 2015;7(3):446-457.

45. Kim RJ, Albert TS, Wible JH, et al. Performance of delayed-enhancement magnetic resonance imaging with gadoversetamide contrast for the detection and assessment of myocardial infarction: an international, multicenter, double-blinded, randomized trial. Circulation. 2008; 117(5):629-637.

46. Ersoy H, Rybicki FJ. Biochemical safety profiles of gadolinium-based extracellular contrast agents and nephrogenic systemic fibrosis. J Magn Reson Imaging. 2007;26(5):1190-1197.

47. Mahrholdt H, Wagner A, Judd RM, Sechtem U. Assessment of myocardial viability by cardiovascular magnetic resonance imaging. Eur Heart J. 2002;23(8):602-619.

48. Lu M, Cohen MH, Rieves D, Pazdur R. FDA report: ferumoxytol for intravenous iron therapy in adult patients with chronic kidney disease. Am J Hematol. 2010;85(5):315-319.

49. Neuwelt A, Sidhu N, Hu CA, Mlady G, Eberhardt SC, Sillerud LO. Ironbased superparamagnetic nanoparticle contrast agents for MRI of infection and inflammation. AJR Am J Roentgenol. 2015;204(3):302-313.

50. Richards JM, Semple SI, MacGillivray TJ, et al. Abdominal aortic aneurysm growth predicted by uptake of ultrasmall superparamagnetic particles of iron oxide: a pilot study. Circ Cardiovasc Imaging. 2011; 4(3):274-281.

51. Sadat U, Howarth SP, Usman A, Tang TY, Graves MJ, Gillard JH. Sequential imaging of asymptomatic carotid atheroma using ultrasmall superparamagnetic iron oxide-enhanced magnetic resonance imaging: a feasibility study. J Stroke Cerebrovasc Dis. 2013;22(8):271-276.
52. Hola K, Markova Z, Zoppellaro G, Tucek J, Zboril R. Tailored functionalization of iron oxide nanoparticles for MRI, drug delivery, magnetic separation and immobilization of biosubstances. Biotechnol Adv. 2015;33(6 Pt 2):1162-1176.

53. Veiseh O, Gunn JW, Zhang M. Design and fabrication of magnetic nanoparticles for targeted drug delivery and imaging. Adv Drug Deliv Rev. 2010;62(3):284-304.

54. Schleich N, Danhier F, Preat V. Iron oxide-loaded nanotheranostics: major obstacles to in vivo studies and clinical translation. J Control Release. 2015;198:35-54.

55. Quyen Chau ND, Menard-Moyon C, Kostarelos K, Bianco A. Multifunctional carbon nanomaterial hybrids for magnetic manipulation and targeting. Biochem Biophys Res Commun. 2015;468(3):454-462.

56. Mura S, Nicolas J, Couvreur P. Stimuli-responsive nanocarriers for drug delivery. Nat Mater. 2013;12(11):991-1003.

57. Wilson WR, Hay M. Targeting hypoxia in cancer therapy. Nat Rev Cancer. 2011;11(6):393-410.

58. Prabhakar U, Maeda H, Jain RK, et al. Challenges and key considerations of the enhanced permeability and retention effect for nanomedicine drug delivery in oncology. Cancer Res. 2013;73(8):2412-2417.

59. National Institutes of Health. Safety and efficacy of doxorubicin adsorbed to magnetic. Beads vs IV doxorubicin in treating liver cancer. ClinicalTrials.gov. Available from: https://clinicaltrials.gov/ct2/ show/NCT00034333. Accessed April 25, 2002, May 23, 2005, May 6, 2016.

60. Cherry EM, Eaton JK. A comprehensive model of magnetic particle motionduring magnetic drug targeting. Int J Multiph Flow. 2014;59: 173-185.

61. Haverkort J, Kenjeres S, Kleijn C. Computational simulations of magnetic particle capture in arterial flows. Ann Biomed Eng. 2009; 37(12):2436-2448.

62. Robey TE, Saiget MK, Reinecke H, Murry CE. Systems approaches to preventing transplanted cell death in cardiac repair. $J$ Mol Cell Cardiol. 2008;45(4):567-581.

63. Cheng K, Li TS, Malliaras K, Davis DR, Zhang Y, Marban E. Magnetic targeting enhances engraftment and functional benefit of iron-labeled cardiosphere-derived cells in myocardial infarction. Circ Res. 2010;106(10):1570-1581.

64. Cheng K, Malliaras K, Li TS, et al. Magnetic enhancement of cell retention, engraftment, and functional benefit after intracoronary delivery of cardiac-derived stem cells in a rat model of ischemia/reperfusion. Cell Transplant. 2012;21(6):1121-1135.

65. Zhang Y, Li W, Ou L, et al. Targeted delivery of human VEGF gene via complexes of magnetic nanoparticle-adenoviral vectors enhanced cardiac regeneration. PLoS One. 2012;7(7):e39490.

66. Li W, Ma N, Ong LL, et al. Enhanced thoracic gene delivery by magnetic nanobead-mediated vector. J Gene Med. 2008;10(8):897-909.

67. Li W, Nesselmann C, Zhou Z, et al. Gene delivery to the heart by magnetic nanobeads. J Magn Magn Mater. 2007;311(1):336-341.

68. Huang Z, Shen Y, Pei N, et al. The effect of nonuniform magnetic targeting of intracoronary-delivering mesenchymal stem cells on coronary embolisation. Biomaterials. 2013;34(38):9905-9916.

69. Huang Z, Shen Y, Sun A, et al. Magnetic targeting enhances retrograde cell retention in a rat model of myocardial infarction. Stem Cell Res Ther. 2013;4(6):149.

70. Vandergriff AC, Hensley TM, Henry ET, et al. Magnetic targeting of cardiosphere-derived stem cells with ferumoxytol nanoparticles for treating rats with myocardial infarction. Biomaterials. 2014; 35(30):8528-8539.

71. Moon H, Park HE, Kang J, et al. Noninvasive assessment of myocardial inflammation by cardiovascular magnetic resonance in a rat model of experimental autoimmune myocarditis. Circulation. 2012; 125(21):2603-2612.

72. Christen T, Nahrendorf M, Wildgruber M, et al. Molecular imaging of innate immune cell function in transplant rejection. Circulation. 2009; 119(14):1925-1932. 
73. Dash R, Chung J, Chan T, et al. A molecular MRI probe to detect treatment of cardiac apoptosis in vivo. Magn Reson Med. 2011;66(4): 1152-1162.

74. Nacev A, Komaee A, Sarwar A, et al. Towards control of magnetic fluids in patients: directing therapeutic nanoparticles to disease locations. IEEE Control Syst Mag. 2012;32(3):32-74.

75. Muthana M, Kennerley AJ, Hughes R, et al. Directing cell therapy to anatomic target sites in vivo with magnetic resonance targeting. Nat Commun. 2015;6:8009.
76. Setsompop K, Kimmlingen R, Eberlein E, et al. Pushing the limits of in vivo diffusion MRI for the Human Connectome Project. Neuroimage. 2013;80:220-233.

77. Richards JM, Shaw CA, Lang NN, et al. In vivo mononuclear cell tracking using superparamagnetic particles of iron oxide: feasibility and safety in humans. Circ Cardiovasc Imaging. 2012;5(4):509-517.

International Journal of Nanomedicine

\section{Publish your work in this journal}

The International Journal of Nanomedicine is an international, peerreviewed journal focusing on the application of nanotechnology in diagnostics, therapeutics, and drug delivery systems throughout the biomedical field. This journal is indexed on PubMed Central, MedLine, CAS, SciSearch $®$, Current Contents $® /$ Clinical Medicine,

\section{Dovepress}

Journal Citation Reports/Science Edition, EMBase, Scopus and the Elsevier Bibliographic databases. The manuscript management system is completely online and includes a very quick and fair peer-review system, which is all easy to use. Visit http://www.dovepress.com/ testimonials.php to read real quotes from published authors.

Submit your manuscript here: http://www.dovepress.com/international-journal-of-nanomedicine-journal 